\title{
Detecção do Molhamento Foliar por meio de Análise de Imagens
}

\author{
Mauricio A. Z. Karrei ${ }^{1}$, Renato W. Dallagasperina ${ }^{1}$, Thiago Benvegnú ${ }^{1}$, \\ Rafael Rieder $^{1}$, Carlos A. Hölbig ${ }^{1}$, José Maurício C. Fernandes ${ }^{1}$, Willingthon Pavan ${ }^{1}$ \\ ${ }^{1}$ Instituto de Ciências Exatas e Geociências - Universidade de Passo Fundo (UPF) \\ 99.052-900 - Passo Fundo - RS - Brasil \\ \{122962, 71429, 105615, rieder, holbig, jmauricio, pavan\}@upf.br
}

\begin{abstract}
This paper presents the implementation of a leaf wetness detection system. Through the use of techniques for analysing and processing of digital images, we sought to identify and quantify the leaf wetness, as well as the leaf wetness duration. Moreover, a wireless sensor network was built to collect variables as temperature, relative humidity and precipitation. Preliminary tests demonstrated the viability and efficiency of the system, highlighting the use of NDVI images to enhance the quantification process. This will allow the correlation between the collected data in order to obtain results with higher accuracy.
\end{abstract}

Resumo. Este trabalho apresenta a implementação de um sistema capaz de detectar o molhamento foliar. Por meio da utilização de técnicas de análise e processamento de imagens digitais, buscou-se identificar e quantificar o molhamento foliar, bem como o tempo de duração deste. Além disso, foi construída uma rede de sensores sem fio capaz de coletar variáveis de temperatura, umidade relativa e precipitação. Testes preliminares comprovaram a viabilidade $e$ a eficiência do sistema, destacando o uso de imagens NDVI para potencializar o processo de quantificação. Isso permitirá a correlação de informações, visando a obtenção de resultados com maior precisão.

\section{Introdução}

A produção de alimentos é um dos grandes desafios mundiais na atualidade, e a agricultura é peça chave para a superação desses desafios. Desta forma, é de fundamental importância a implementação de novas tecnologias e ferramentas que otimizem o uso de insumos agrícolas e o manejo das culturas, contribuindo para a redução dos impactos ambientais, aumento da produtividade e, consequentemente, aumentando a lucratividade do produtor [Greenwade 1993].

A prática da agricultura automatizada e baseada em sensores, faz parte da agricultura de precisão, e está crescendo a cada dia, porém, seu avanço passa por questões como custo de implementação, resistência dos equipamentos em ambientes agressivos, manutenção e fornecimento de energia aos dispositivos eletrônicos em locais de difícil acesso [Rehman et al. 2014].

A germinação e a esporulação de fungos, bem como a infecção das plantas, são diretamente afetadas por fatores como a temperatura e a umidade do ar, a ação do vento e o molhamento foliar. Dentre estes parâmetros, o período do molhamento foliar, que 
pode ser causado pela chuva, neblina, irrigação ou orvalho [Sentelhas et al. 2008], é um dos que mais impactam na relação entre o fungo e a planta, tornando esse um parâmetro de grande importância em modelos de simulação de doenças de plantas. Existem muitas formas de se realizar esta detecção, porém, algumas são falhas e outras são caras, além de existirem lacunas com relação a quantificação e normalização dos dados coletados [Rowlandson et al. 2015].

Nos últimos anos foram desenvolvidas diversas tecnologias na busca pelo monitoramento da duração do molhamento foliar (DMF) nas mais diferentes culturas [?]. Apesar dos resultados se mostrarem positivos, ainda há barreiras a serem superadas para ampliar a utilização deste artifício. Partindo do princípio de que um dos métodos mais utilizados e confiáveis para a determinação da DMF é a observação humana, é possível considerar que a visão computacional, por ser um método semelhante, é uma possibilidade interessante a ser explorada.

Entre os diversos dispositivos existentes no mercado, é possível destacar os sensores eletrônicos, que são amplamente utilizados. Estes sensores, simulam a folha e são capazes de detectar a presença de água em sua superfície, podendo ser resistivos [Inc. 2010] ou capacitivos [Devices 2010]. A implementação de sensores de umidade e temperatura, que possibilitam estimar o molhamento foliar por meio de modelos empíricos, também apresenta-se como uma das formas mais viáveis para a determinação da duração do molhamento foliar [Durigon and van Lier 2013, Leandro et al. 2003].

Estas tecnologias costumam ser suscetíveis a variações, de acordo com o tipo de planta analisado e as condições climáticas do local em que estão instalados. Os sensores de molhamento exigem cuidados como ângulo de instalação, posicionamento e diferença das características termodinâmicas entre as superfícies do sensor e da folha, enquanto os modelos empíricos necessitam de calibração e ajustes nos limiares de parâmetros de interesse de acordo com as características do local de análise e a espécie de planta monitorada [Rowlandson 2011].

Nos últimos anos foram desenvolvidas diversas tecnologias na busca pelo monitoramento do molhamento foliar nas mais diferentes culturas [Sentelhas 2004]. Apesar dos resultados se mostrarem positivos, ainda existem barreiras a serem superadas para ampliar a utilização deste artifício. Partindo do princípio de que um dos métodos mais utilizados e confiáveis para a determinação do molhamento foliar é a observação humana, é possível considerar que a visão computacional, por ser um método semelhante, é uma possibilidade que deve ser explorada.

Devido aos constantes avanços tecnológicos, a captura e processamento remoto de imagens está se tornando cada vez mais acessível. O processamento de imagens apresenta vantagens em relação ao uso de sensores convencionais, como por exemplo o armazenamento das imagens capturadas para posterior análise e validação dos resultados, aumentando a confiabilidade nos dados gerados.

Assim, diante das informações expostas, é plausível considerar que o desenvolvimento de um sistema capaz de coletar imagens e variáveis ambientais, utilizando o processamento destas imagens e informações para determinar a presença de molhamento foliar oferece diversos benefícios a profissionais da área agrícola. Com isso, o objetivo deste trabalho é apresentar o desenvolvimento de um sensor capaz de detectar o molha- 
mento foliar por meio de análise de imagens, além de uma rede de sensores sem fio de baixo custo, com o intuito de auxiliar no manejo de doenças de plantas.

\section{Material e Métodos}

Nesta sessão são abordados os conceitos fundamentais para a plena compreensão do projeto. São apresentadas as ferramentas de hardware utilizadas, bem como as bibliotecas de software que compuseram o desenvolvimento da solução.

\subsection{Estrutura Geral do Sistema}

Como peça principal para a realização deste trabalho, foi utilizado a placa de desenvolvimento Intel Galileo Gen2 (Figura 1(a)). Esta placa, é responsável por realizar as tarefas de coleta e processamento de imagens, bem como a aquisição dos dados provenientes da Rede de Sensores Sem Fio (Seção 2.4).

Para a captura das imagens utilizadas na identificação do molhamento foliar, é utilizada uma câmera com comunicação USB (Figura 1(d)), escolhida por sua compatibilidade com a placa Galileo.

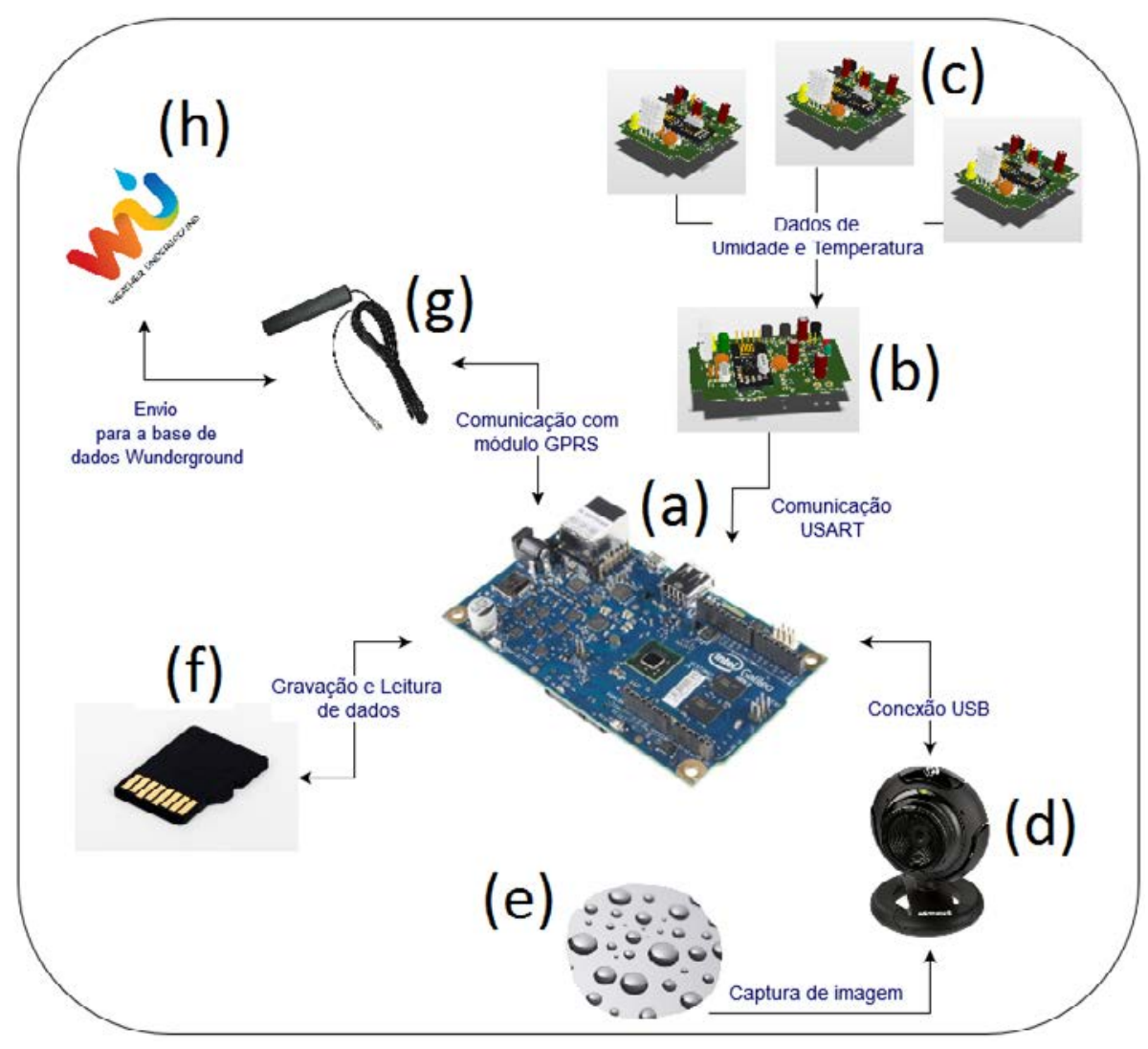

Figura 1. Diagrama em blocos do sistema proposto.

\subsection{Captura de imagens}

Para a realização da coleta das imagens, a câmera USB conectada à Galileo é ativada sempre que os dados coletados pela rede de sensores (Seção 2.4) indicam a probabilidade de existência do molhamento foliar. 
O ambiente onde as imagens são capturadas, influência diretamente no resultado do processamento digital destas. Muitas são as variáveis presentes no ambiente com potencial de interferência sobre o processo, algumas das quais podem ser citadas: iluminação, reflexo, cores de fundo, presença de movimento, ângulo da captura da imagem, entre outros. Para testes e validação inicial do sistema foi desenvolvido um ambiente controlado para minimizar a influência de algumas destas variáveis. Na Figura 2 é exibido o ambiente controlado construído.

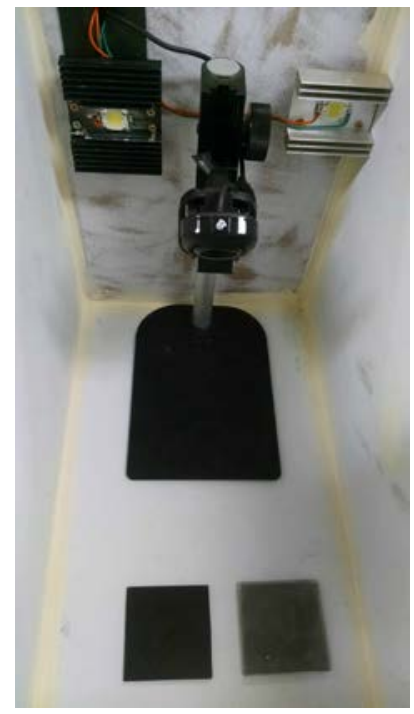

Figura 2. Ambiente controlado construído para captura de imagens.

\subsection{Superfície Alternativa}

O ambiente natural possui grande influência sobre o resultado do processamento das imagens. Dessa forma, para eliminar o ruído causado pelo movimento da folha e pelos ruídos de fundo da imagem buscou-se uma superfície alternativa à folha da planta. Esta superfície deveria ser fixa, ser capaz de acumular água e ser suficientemente robusta para suportar condições adversas. Neste projeto, utilizou-se como superfície alternativa um fragmento de vidro jateado, onde sua opacidade com superfície seca, permite uma leitura mais próxima da cor branca, e no instante que a superfície possui acúmulo de água, o vidro jateado apresenta maior transparência.

Dessa forma, utilizou-se um material de cor preta colocado sob o vidro jateado. Assim, no momento em que ocorrer a presença de água livre sobre a superfície do vidro jateado, tornará visível o material posicionado sob o vidro. Caso a superfície do vidro não contenha água, a cor preta não terá tanto destaque e consequentemente não será identificada durante o processamento da imagem. Na Figura 3 é apresentado um fragmento do vidro utilizado. Nota-se que há um maior destaque da cor preta apenas na região com concentração de água.

\subsection{Rede de Sensores sem Fio}

Todos os nodos da rede de sensores sem fio possuem hardware idêntico, diferenciandose apenas pela função exercida e pelo firmware embarcado. O nodo coordenador (Figura 1(b)) é responsável pelo recebimento dos dados coletados pelos demais nodos da 


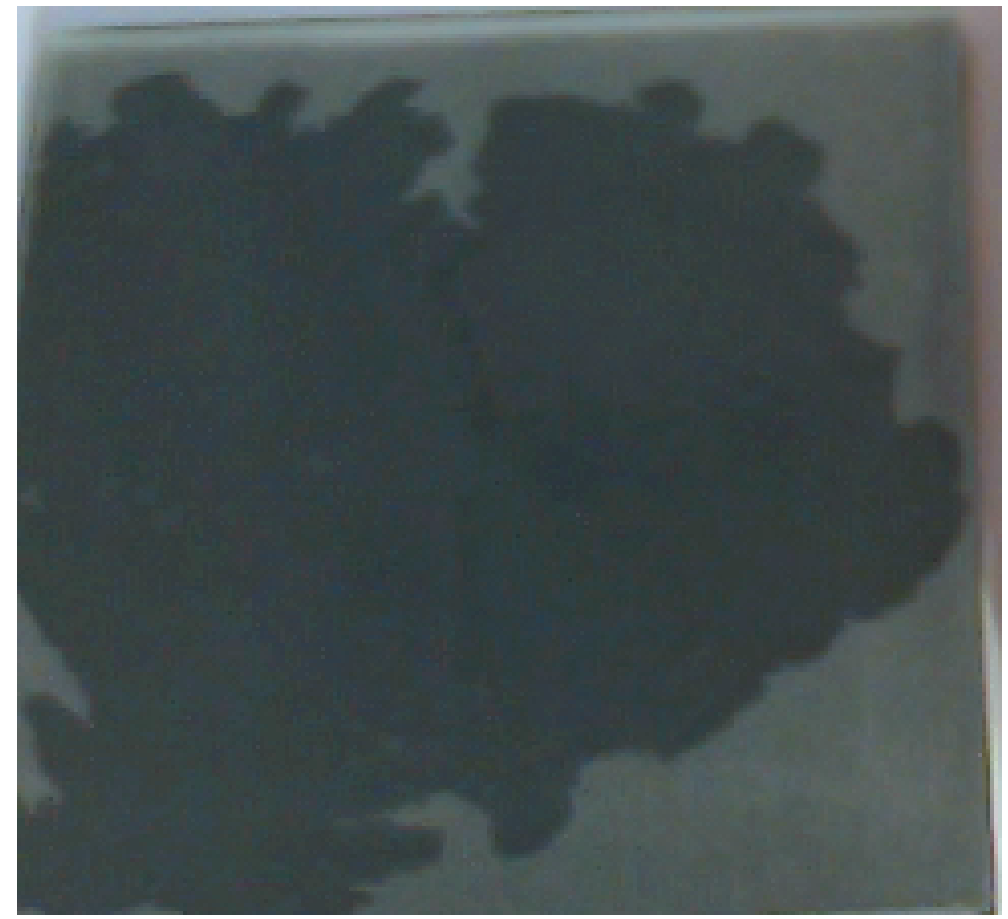

Figura 3. Superfície do vidro jateado, ressaltando a área molhada.

rede (Figura 1(b)), e transmissão destes dados para a Galileo. Cada nodo da rede possuí sensores de temperatura e umidade relativa do ar.

Para a coleta de dados e também para aumentar a área de abrangência do sistema, foi desenvolvida uma rede de sensores sem fio. Todos os nodos são alimentados por baterias e células fotovoltaicas, garantindo sua autonomia energética. A rede utiliza a topologia estrela, sendo o nodo coordenador instalado junto a Galileo. O nodo coordenador possui, além do sensor de temperatura e umidade relativa do ar um sensor de precipitação.

\subsection{Plataforma de recebimento de dados}

Weather Underground ou Wunderground é um concentrador e fornecedor de dados meteorológicos, formado principalmente por estações meteorológicas particulares. Qualquer pessoa ou instituição pode enviar os dados coletados, em tempo real, para o Wunderground. Segundo informações do próprio serviço, atualmente, somente nos Estados Unidos existem mais de cem mil estações enviando informações, sendo mais de oitenta mil particulares, de "hobbystas"ou entusiastas da área.

Assim, uma alternativa para disponibilizar as informações coletadas por este projeto ao público foi a utilização dos serviços oferecidos pelo Wunderground.

É utilizado um módulo GPRS, Figura 1(g), para o envio dos dados coletados à uma plataforma responsável pelo armazenamento, processamento e tratamento destes dados, também pode ser utilizado para enviar alertas via SMS. A plataforma usada para receber e disponibilizar os dados coletados ao publico é a Weather Underground [und 2015], Figura 1(h). E ainda, todos os dados e imagens coletados são armazenados em um SD Card, Figura 1(f), ficando disponíveis para acesso quando necessário. A Figura 1 mostra o diagrama completo do sistema desenvolvido. 


\subsection{OpenCV}

O processo de identificação do molhamento foliar, deu-se por meio da captura e processamento de imagens, utilizando-se de uma câmera USB conectada à plataforma Galileo. No que se refere ao método de análise e identificação da presença de água na estrutura montada (vidro jateado), foram realizados testes utilizando a biblioteca OpenCV.

Open Source Computer Vision Library (OpenCV) é uma biblioteca código fonte aberto que possui um conjunto de funções e recursos, possibilitando a construção de softwares de visão computacional. Nesta biblioteca estão incluídos um conjunto de algoritmos otimizados, que podem ser utilizados em aplicações de detecção e reconhecimento de faces/objetos e manipulação de imagens, tanto em 2D como em 3D [Cunha 2013].

O software foi implementado utilizando a linguagem de programação Python e o seu módulo "cv2", o qual é carregado com a utilização do pacote "python-opencv". Do módulo "cv2", foram utilizadas as funções VideoCapture e inRange para a manipulação das imagens. A primeira consiste em uma função responsável por realizar o interfaceamento do software desenvolvido com a câmera conectada à Galileo, oferecendo recursos para a captura da imagem da câmera. Já na função inRange, estão disponíveis os recursos necessários para analisar as imagens capturadas.

\subsection{Armazenamento dos Dados Coletados}

As informações provindas dos sensores de umidade relativa do ar, temperatura, precipitação, molhamento foliar, resultado do processamento da imagem e horário da coleta são transmitidas ao concentrador, e este, por sua vez, envia os dados para a Web por meio de uma conexão GPRS/3G ou por meio de uma conexão ethernet.

As imagens capturadas pelo sistema, além de serem processadas e analisadas, também são armazenadas em um cartão SD juntamente com os dados capturados pelos sensores, assim como informações do horário da coleta. Desta forma, quando necessário, o usuário pode acessar todas as informações diretamente do cartão SD.

\section{Resultados}

\subsection{Rede de Sensores sem Fio}

Os protótipos dos nodos da rede foram projetados e montados visando baixo custo e baixo consumo. Todos os nodos possuem hardware idêntico, diferenciando-se apenas pelo firmware embarcado. Para minimizar o consumo de energia, o sistema opera de forma que o nodo coordenador se mantém em modo de recepção constantemente, enquanto os demais nodos operam em modo sleep, despertando apenas para a coleta e envio de dados.

O sensor de temperatura e umidade relativa do ar precisou ser acomodado em uma estrutura especial, onde não ficasse exposto diretamente a luz solar, evitando que esta altere o valor lido pelo sensor. Na Figura 4 é apresentado o protótipo de um nodo da rede, com toda a estrutura necessária. 


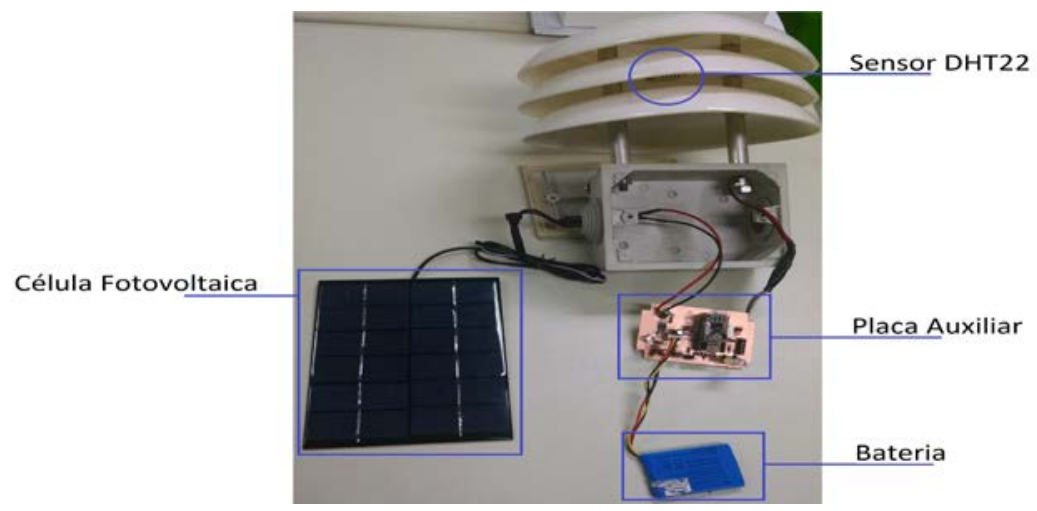

Figura 4. Nodo da rede contendo todos os componentes utilizados.

\subsection{Interpretação de Imagens}

\subsubsection{Detecção de Bordas}

Inicialmente, realizaram-se testes utilizando técnicas de processamento de imagens para detecção de bordas, utilizando o método Canny Edge Detector da biblioteca OpenCv. O objetivo deste processo foi capturar a formação de água nas folhagens de plantas a partir da identificação do formato de sua borda na superfície da folha.

O uso desta técnica foi descartada para utilização neste projeto, visto que não apresentou bons resultados para a detecção da borda da gota d'água na superfície da folha. Devido a aspectos naturais do formato das folhas das plantas e também devido ao nível de transparência da água, nota-se que após a aplicação dos filtros de detecção de borda, o nível de falsa detecção obteve grande proporção. A Figura 5 apresenta o resultado da aplicação da técnica de detecção de borda.
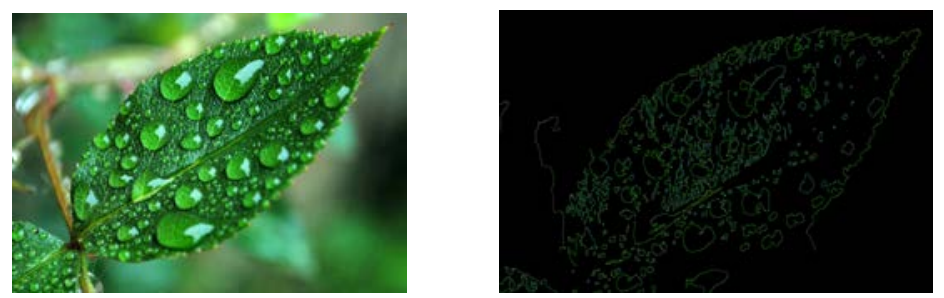

Figura 5. Identificação de formação de gotículas d'água utilizando técnicas de detecção de bordas.

\subsubsection{Detecção de intervalos de cores}

Em razão da dificuldade em realizar a detecção de água por meio da técnica de detecção de bordas, optou-se por utilizar a técnica de filtragem de cores. Para isso, a partir da obtenção da imagem da câmera USB no formato RGB, esta é convertida para o formato HSV com o auxílio da função da biblioteca OpenCV cvtColor. Esta conversão permite que os frames coletados sejam aplicados na função inRange, a qual recebe como parâmetro três valores: 1) frame coletado (imagem no formato HSV) e realiza o thresholding na imagem; 2) array com o intervalo baixo de cores (menor tonalidade da cor preta) a serem filtradas 
na imagem; e 3) array contendo o intervalo máximo de cores (maior tonalidade da cor preta) a serem filtradas na imagem. O retorno desta função é uma imagem contendo a identificação dos pixels que contém a cor preta e também dos pixels que não contém a cor preta.

Este método de análise apresentou maior eficácia em comparação com o método de detecção de bordas, visto que as interferências na superfície da folha da planta, encontradas no primeiro método, ficaram inexistentes neste segundo método. Em consequência disso, foi possível identificar a presença de água na superfície do vidro, visto que no caso de existência de água, o vidro perde sua opacidade e ganha com um aspecto "transparente", tornando visível a cor preta que se encontra por detrás do vidro, permitindo assim, tornar viável a identificação de água sobre a superfície do vidro jateado. Esta identificação, é apresentada na Figura 6.
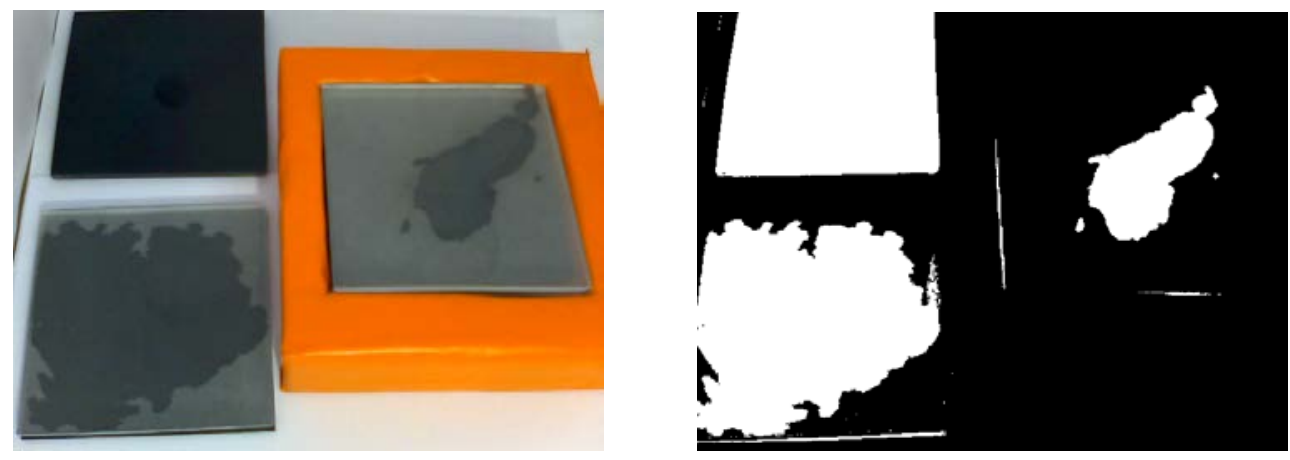

Figura 6. Processo de identificação da concentração de água no vidro jateado.

Além do exposto, o software desenvolvido realiza a quantificação de pixels, sendo possível, dessa forma, quantificar os pixels que são formados pela cor preta e os pixels formados por cores diferentes de preto.

\subsection{Transmissão de dados}

Para a transmissão dos dados coletados foram utilizados dois meios de comunicação: um via GPRS (telefonia móvel) e outro por meio de uma conexão Ethernet, sendo que ambos se mostraram eficazes. Os dados foram transmitidos para a plataforma Weather Underground [und 2015].

\section{Discussões e Conclusões}

A partir dos resultados apresentados, percebe-se que a identificação de molhamento foliar por meio de análise de imagens pode ser realizada. A superfície do vidro jateado, para esta situação, foi utilizada como alternativa à folha da planta e apresentou bons resultados.

Com a realização dos experimentos, constatou-se que fatores externos como intensidade de iluminação, ângulo da câmera e distância da câmera exercem forte influência sobre o funcionamento do sistema. Para tanto, nota-se que é preciso, primeiramente, dominar esses fatores em um ambiente controlado, para posterior aplicação e ajustes em ambiente aberto.

A quantificação do molhamento foliar por meio do processamento de imagens mostrou-se viável e eficiente. Além dos resultados obtidos, um novo trabalho está sendo 
realizado, adaptando-se a câmara para funcionar com um filtro NDVI (Normalized Difference Vegetation Index [INSA 2008]). A Figura 7 apresenta alguns resultados preliminares obtidos utilizando-se o método do NDVI. Pode-se observar que a área das folhas da planta que possuem acúmulo de água ficaram destacadas em um tom de verde. As áreas onde existe acúmulo de água, mas não estão sobre a superfície das folhas da planta, obtiveram uma coloração na tonalidade azul. Além disso, nota-se que as áreas das folhas da planta onde não existe acúmulo de água, a cor vermelha e/ou amarela prevaleceu.

Este método também apresenta potencialidade de utilização para a identificação e quantificação do molhamento foliar. Com isso, destaca-se como fator positivo, a possibilidade da identificação da presença de água na superfície das plantas sendo feita diretamente no ambiente real, sem a utilização superfícies alternativas a folha das plantas.
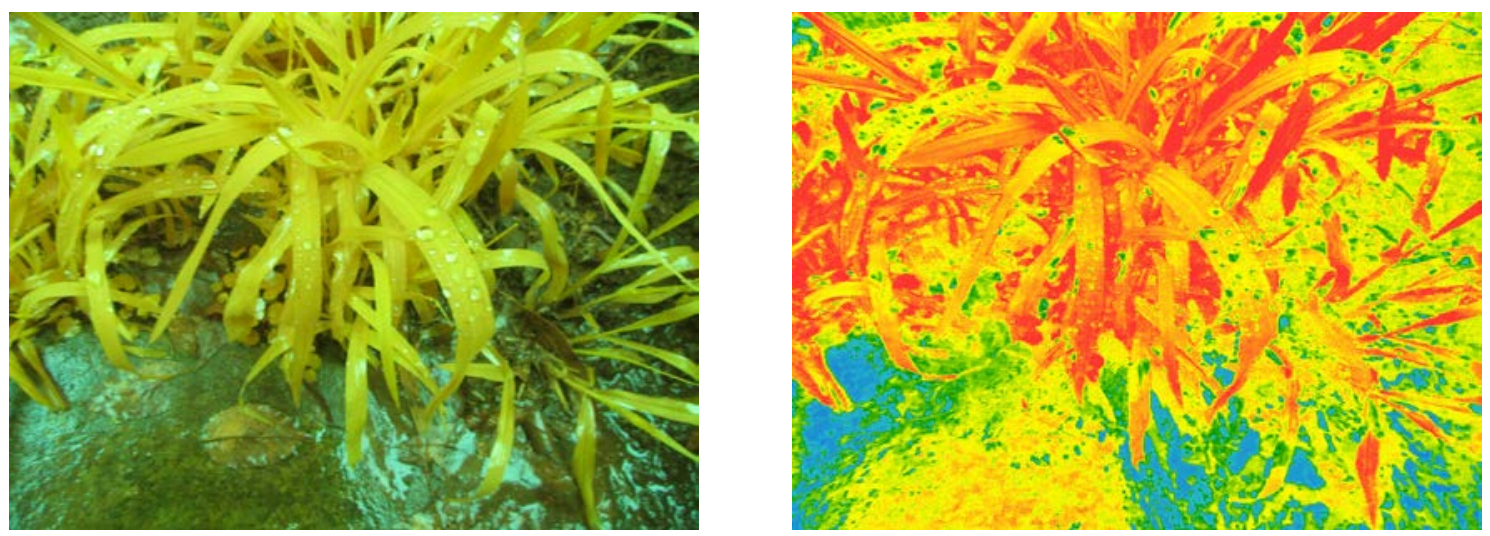

Figura 7. Imagens capturadas utilizando filtro NDVI.

Com o desenvolvimento deste trabalho, uma nova forma de identificar e quantificar o molhamento foliar foi determinada. Os resultados indicam que o uso do processamento digital de imagens é uma alternativa viável aos sensores de molhamento foliar presentes no mercado atualmente. Além disso, a utilização da rede de sensores sem fio permite extrair diferentes variáveis do ambiente, possibilitando a correlação de informações (como umidade do ar) a fins de validar e garantir maior precisão para a detecção do molhamento foliar.

\section{Referências}

(2015). Weather underground.

Cunha, A. L. B. N. d. (2013). Sistema automático para obtenção de parâmetros do tráfego veicular a partir de imagens de vídeo usando OpenCV. $\mathrm{PhD}$ thesis, Universidade de São Paulo.

Devices, D. (2010). Leaf wetness dielectric sensor. Technical report.

Durigon, A. and van Lier, Q. (2013). Duração do período de molhamento foliar: Medição e estimativa em feijão sob diferentes tratamentos hídricos. Rev Bras Eng Agric Ambient, 17:200-207.

Greenwade, G. D. (1993). The Comprehensive Tex Archive Network (CTAN). TUGBoat, 14(3):342-351. 
Inc., C. S. (2010). Model 237 leaf wetness sensor. Technical report.

INSA, I. N. d. S. (2008). Índice de vegetação por diferença normalizada.

Leandro, L., Gleason, M., Nutter Jr, F., Wegulo, S., and Dixon, P. (2003). Influence of temperature and wetness duration on conidia and appressoria of colletotrichum acutatum on symptomless strawberry leaves. Phytopathology, 93(4):513-520.

Rehman, A., Abbasi, A. Z., Zubair, N. I., and Shaikh, A. (2014). A review of wireless sensors and networks applications in agriculture. Computer Standards and Interfaces, 36:263-270.

Rowlandson, T., Gleason, M., Sentelhas, P., Gillespie, T., Thomas, C., and Hornbuckle, B. (2015). Reconsidering leaf wetness duration determination for plant disease management. Plant Disease, 99(3):310-319.

Rowlandson, T. L. (2011). Leaf wetness: implications for agriculture and remote sensing.

Sentelhas, P. C. (2004). Duração do período de molhamento foliar: aspectos operacionais da sua medida, variabilidade espacial em diferentes culturas e sua estimativa a partir do modelo de penman-monteith. Piracicaba: ESALQ/USP (Tese de Livre-Docência).

Sentelhas, P. C., Marta, A. D., Orlandini, S., Santos, E. A., Gillespie, T. J., and Gleason, M. L. (2008). Suitability of relative humidity as an estimator of leaf wetness duration. Agricultural and forest meteorology, 148:392-400. 